\title{
Coherent Control of Light Absorption and Carrier Dynamics in Semiconductor Nanostructures
}

\author{
WALTER PÖTZ* and XUEDONG HU \\ University of Illinois at Chicago, Physics Department, Chicago, IL 60607, USA
}

\begin{abstract}
We present two examples of coherent control of inter(sub)band transitions in a semiconductor double weil by coherent light sources. Accounting for the upper hole subband and two lowest electron subbands, a microscopic theoretical analysis shows that electron-hole pair generation by a sub-picosecond pump pulse can be controlled by the intensity and the phase of a dc microwave field which resonantly couples the two electron subbands. Light absorption can be either enhanced or reduced. Secondly, it is shown that proper combination of two pulsed laser fields allows control of electron inter(sub)band transitions and final-state population, i.e., the formation of indirect versus direct excitons.
\end{abstract}

Keywords: Coherent control, semiconductor, quantum well, optics, theory

Coherent control of final-state population and chemical reactions has long been pursued in atomic and molecular physics [1-5]. More recently, improvements in ultrafast spectroscopy have allowed induction and observation of coherent phenomena in semiconductors, in form of coherent charge oscillations in double wells, Bloch oscillations, and coherent control of photocurrent, to name some of the highlights of progress in recent years [6-10]. In this paper we investigate coherent control of inter(sub)band transitions, absorption, and final-state population theoretically. Specifically we discuss a scheme which allows phase controlled light absorption and control of final-state population in semiconductor heterostructures by the interplay of two coherent light sources. As a specific example we give results for an asymmetric $145 \AA / 25 \AA / 100 \AA$ GaAs-AlGaAs-GaAs double well whose electron subband splitting is controlled by a static electric field and which is exposed to a tunable sub-picosecond pump pulse which generates electron-hole pairs (excitons) across its main energy gap. Calculations are done within the framework of a microscopic theory in form of Boltzmann-Bloch equations which account for the carrier-carrier Coulomb interaction [11]. In the present study, subband splittings and time scales are such that LO phonon

* Corresponding author. 
scattering may be neglected. Light pulses are of Gaussian shape and the light-matter coupling is treated for a classical light field including the counter-rotating part. The peak of the pump pulse arrives at time zero, relative to which we define the phase $\phi$ of the microwave (MW) field $\vec{E}_{\mathrm{MW}}(t)=$ $\vec{E}_{0}(t) \cos \left(\omega_{\mathrm{MW}} t+\phi\right)$. A comparison to the rotating-wave approximation (RWA), which has been used in an earlier study, is made [12].

When a coherent dc MW field resonantly couples the two electron subbands, the system undergoes Rabi oscillations between its uncoupled eigenstates when originally prepared in one of the latter. In the situation depicted in Figure 1, the dipole matrix element between hole $|1\rangle$ and upper electron subband $|2\rangle$ is much stronger than hole and lower electron subband $|3\rangle$. Hence, if a pump pulse is applied at resonance with the direct exciton maximum absorption is obtained. However, application of a MW field reduces the admixture of left-well eigenfunction $|W\rangle$ in $|2\rangle$ and transfers it to $|3\rangle$. Hence, the MW field reduces absorption at the direct exciton peak [13]. Conversely, if the pump pulse is tuned near the indirect exciton, application of a MW field enhances

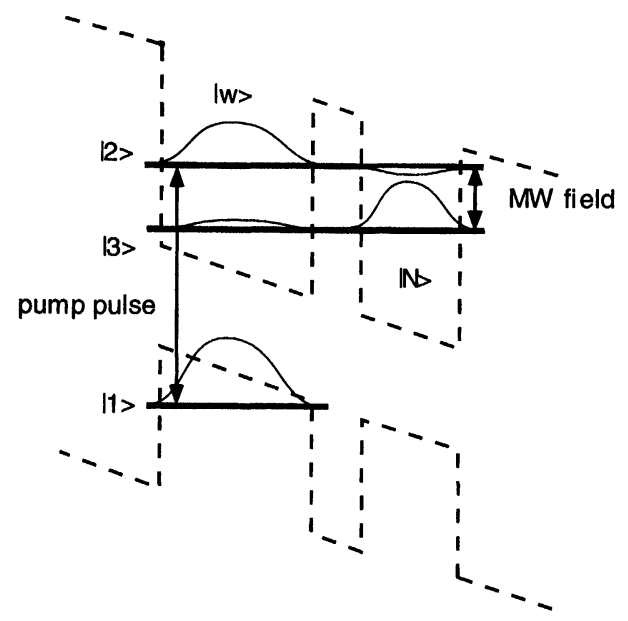

FIGURE 1 Illustration of a biased asymmetric GaAlAsGaAs double well. $|1\rangle,|2\rangle$ and $|3\rangle$ denote double-well eigenstates; $|W\rangle$ and $|N\rangle$ are the lowest wide-well and narrow-well eigenstates, respectively. The shape of wave functions is indicated by thin solid lines on top of the corresponding energy levels. absorption. The phase of the coherent dc MW field enters the coupling between the electron subbands and hence the complex electron interband polarization. When the pump pulse duration is shorter than the inverse of the MW-induced Rabi frequency, the phase influences the absorption process from a third level, here, the top hole subband $|1\rangle$. This is demonstrated in Figure 2 for the case where a 80 fs pump pulse of Gaussian profile is tuned near resonance with the indirect excitons of the DW with a subband splitting of $20 \mathrm{meV}$. The $\mathrm{MW}$ intensity is about $2 \mathrm{MWcm}^{-2}$, corresponding to a Rabi period near $100 \mathrm{fs}$. Clearly, the presence of the MW field increases absorption by about 15 percent for phase $\pi / 2$. This effect is more pronounced at the direct exciton peak [13] The RWA underestimates the importance of second harmonics in the carrier dynamics induced by the MW field. Therefore, shorter pump pulses than predicted by within the RWA may be required to display the phase dependence of absorption.

For the present three-level system and within the RWA for transitions between conduction and valence bands, the pump pulse photon Boltzmann equation in the presence of coherence in the carrier

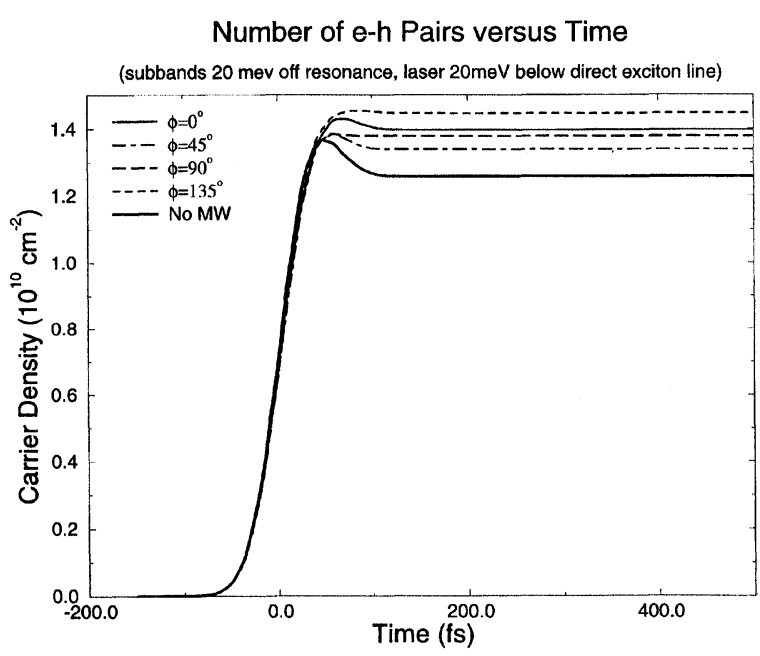

FIGURE 2 Density of photo-generated electron-hole pairs as a function of time and MW phase $\Phi$ for a pump pulse photon energy which is $20 \mathrm{meV}$ below the direct exciton peak. 
system is of the structure [11].

$$
\begin{aligned}
\frac{d N(q, t)}{d t}= & \frac{2 \pi}{\hbar^{2}} \sum_{k, \alpha, \alpha^{\prime}=2,3} M_{1 \alpha^{\prime}}(q) M_{1 \alpha}^{*}(q) \delta_{E} \\
& \left\{f_{\alpha \alpha^{\prime}}(k, t)\left(1-f_{11}(k, t)\right)(N(q, t)+1)\right. \\
& \left.-f_{11}(k, t)\left(1-f_{\alpha \alpha^{\prime}}(k, t)\right) N(q, t)\right\}, \\
\approx & \frac{2 \pi}{\hbar^{2}} N(q, t) \sum_{k, \alpha, \alpha^{\prime}=2,3} M_{H \alpha^{\prime}}(q) M_{H \alpha}^{*}(q) \delta_{E} \\
& \left\{f_{\alpha \alpha^{\prime}}(k, t)-f_{11}(k, t)\right\}, \text { for } N(q, t) \gg 1,
\end{aligned}
$$

where $f_{\alpha \alpha^{\prime}}(k, t)$ are carrier distribution functions, for $\alpha=\alpha^{\prime}$, and interband polarizations, for $\alpha \neq \alpha^{\prime}$. $N(q, t)$ is the photon occupation number and $M_{\alpha \alpha^{\prime}}(q)$ is the matrix element for coupling subband $\alpha$ to subband $\alpha^{\prime}$ via photon $q . \delta_{E}$ is the appropriate energy-conserving delta function. This equation shows that electron interband polarization influences photon absorption. Figure 3, in turn, shows how the phase of the MW field determines the sign of the real part of the electron interband polarization during the presence of the pump pulse to allow coherent control of the absorption process.

A possible scheme to control the final-state population in semiconductors resembles previous work on atoms for which adiabatic switching has been demonstrated to allow transitions between molecular levels which are dipole-forbidden by

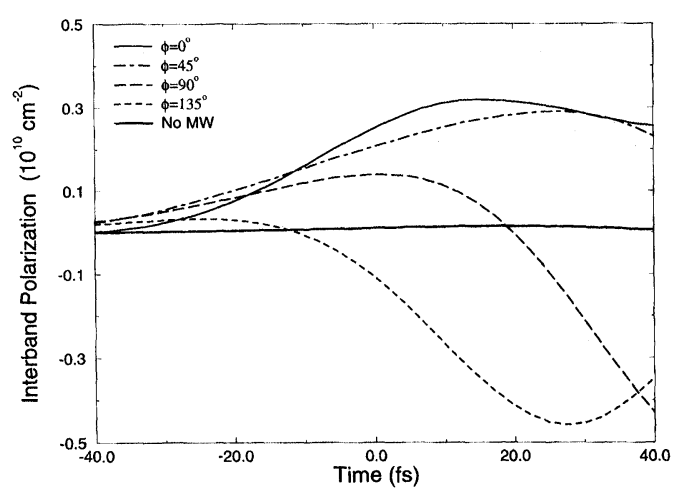

FIGURE 3 Real part of $|W\rangle-|N\rangle$ interband polarization as a function of time and MW phase. means of optical coupling to a third level $[4,5]$. In this scheme, which is sketched in Figure 1, two temporally and spatially overlapping light pulses couple three levels of the system. The initial state of the system is $|1\rangle$, the desired final state is $|3\rangle$, and the intermediate state is $|2\rangle$. If the light pulse coupling $|2\rangle$ and $|3\rangle$, with amplitude $a(t)$ and duration $T_{\mathrm{MW}}$, arrives and ends before arrival and, respectively, end of the pulse coupling $|1\rangle$ and $|2\rangle$, with amplitude $b(t)$ and duration $T_{p}$, and the pulse amplitudes change sufficiently slowly (relative to the characteristic frequencies of the driven threelevel system), stimulated Raman adiabatic passage (STIRAP) occurs and the system undergoes an adiabatic transition from level $|1\rangle$ to level $|3\rangle$. [4] The condition for an adiabatic transition for $T=T_{p}=T_{\mathrm{MW}}$ is

$$
T \cdot\left[\frac{\delta}{2} \pm \sqrt{\frac{\delta^{2}}{4}+|a|^{2}+|b|^{2}}\right] \gg \hbar,
$$

where $\delta$ is the light field detuning.

However, in semiconductors resonant coupling between subbands calls for MW pulses, which may be difficult to generate at sufficiently high intensity. Moreover, due to characteristic decoherencing times for (free) carriers in semiconductors passage times can not be much longer than a picosecond. Therefore, the STIRAP process may be impractical, if not impossible, to be adopted to semiconductor nanostructures. Hence, we have investigated sub-picosecond nonadiabiatic transfer based on the three-subband scheme in Figure 1, where subband $|1\rangle$ is the top hole subband of a heterostructure, $|2\rangle$ and $|3\rangle$ are the two lowest electron subbands separated by $25 \mathrm{meV}$, in the present case. A $100 \mathrm{fs}$ subpicosecond pump pulse is used to generate electron-hole pairs (direct excitons) associated with levels $|1\rangle$ and $|2\rangle$. Its duration must be shorter than the inverse Rabi frequency between subband $|2\rangle$ and $|3\rangle$ which is established by a concurrent MW pulse. For excitation densities of about $10^{10}$ carriers per $\mathrm{cm}^{2}$ this ensures $\mathrm{MW}$-induced charge oscillations between subband $|2\rangle$ and $|3\rangle$. Intensity and 
duration of the MW field are adjusted so that the latter permits one half of a Rabi oscillation between $|2\rangle$ and $|3\rangle$, such that the electrons get trapped in state $|3\rangle(\approx|N\rangle)$ after a single tunneling process. Here, the MW pulse resonantly couples the two electron subbands $(\delta=0)$ and has a duration of $T_{\mathrm{MW}}=320 \mathrm{fs}$, corresponding to about two MW periods, and a peak intensity of about 1

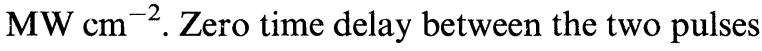
and phase $\pi$ of the MW field relative to the peak of the pump pulse were found to give best results.

In Figure 4 we show the number of electrons in the left well versus the number of electrons in the right well, and the total number of carriers (holes). Calculations including the counter-rotating field contributions, thick lines, are compared to those within the RWA, thin lines. It is clearly evident that the presence of the MW field reverses the tendency for direct (solid lines) versus indirect exciton (dot-dashed lines) formation. Without MW field and owing to the shape of wave functions, predominantly direct excitons are formed initially. Figure 4 also shows that the RWA gives almost quantitatively correct results for the final state population in the present case. However, it predicts simple (damped) harmonic charge oscillations, whereas the full calculation shows a more complicated dynamics.

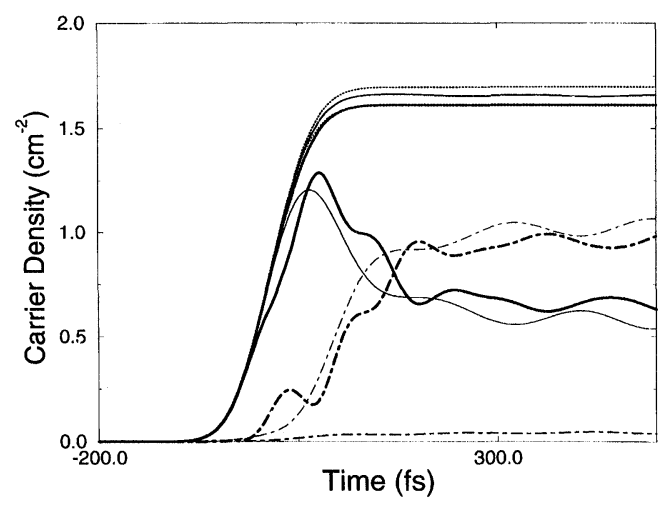

FIGURE 4 Carrier densities in the double well versus time. Solid lines: wide well (direct excitons); dot-dashed lines: narrow well (indirect excitons); dashed lines: holes (total number of excitons); thick lines: with microwave field (phase $\pi$ ) regular lines: no MW field; thin lines: MW field in RWA approximation.
In summary, we have given theoretical results which indicate that coherent control of intersubband transitions on a subpicosecond time scale is possible by means of coherent light sources. In particular, we have investigated coherent control of light absorption and final-state population in semiconductor double wells. It should be pointed out that the latter should also be achievable by interference between single and triple photon absorption, in analogy to experiments on molecules [3], and coherent current control via interference between single and two-photon absorption [8]. Details of these findings will be published elsewhere [12]. The rotating-wave approximation, which allows analytic solution of the coupled three-level system, is found to give nearly quantitatively correct results for the non-adiabatic finalstate-control processes. However, for the coherent control process of absorption discussed here, it merely gives qualitatively correct results. Specifically, it fails to give an accurate account of charge oscillations induced by the MW field and, consequently, the correct value for the phase of optimal coupling.

\section{Acknowledgements}

We thank Prof. R. J. Gordon and Prof. W. A. Schroeder for helpful discussions. This work has been supported by the US Army Research Office.

\section{References}

[1] Tannor, D. J. and Rice, S. A. (1988). Adv. Chem. Phys., 70, 441.

[2] Brumer, P. and Shapiro, M. (1989). Accts. Chem. Res., 22, 407.

[3] Lu, S., Park, S. M., Xie, Y. and Gordon, R. J. (1992). J. Chem. Phys., 96, 6613.

[4] Oreg, J., Hioe, F. T. and Eberly, J. H. (1994). Phys. Rev. $A, 29,690$.

[5] Gaubatz, U., Rudecki, P., Schiemann, S. and Bergmann, K. (1990). J. Chem. Phys., 92, 5363; Bergmann, K. and Shore, B. W. (1995). in Molecular Dynamics and Spectroscopy by Stimulated Emission Pumping, Eds. H.-L. Dai and R. W. Field (World Scientific, Singapore), p. 315.

[6] Heberle, A. P., Baumberg, J. J. and Köhler, K. (1995). Phys. Rev. Lett., 75, 2598. 
[7] Citrin, D. S. (1996). Phys. Rev. Lett., 77, 4596.

[8] Hachè, A., Kostoulas, Y., Atanasov, R., Hughes, J. L. P., Sipe, J. E. and van Driel, H. M. (1997). Phys. Rev. Lett., 78, 306.

[9] Leo, K., Shah, J., Göbel, E. O., Damen, T. C., SchmittRink, S., Schäfer, W. and Köhler, K. (1991). Phys. Rev. Lett., 66, 201.

[10] Waschke, C., Roskos, H. G., Schwedler, R., Leo, K., Kurz, H. and Köhler, K. (1993). Phys. Rev. Lett., 70, 3319.

[11] Pötz, W. (1996). Phys. Rev. B, 54, 5647; Pötz, W. (1996). Appl. Phys. Lett., 68, 2553; Pötz, W. and Hohenester, U., unpublished.

[12] Pötz, W. (1997). Appl. Phys. Lett., 71, 395.

[13] Pötz, W. (1997). Phys. Rev. Lett., 79, 3262.

\section{Authors' Biographies}

Walter Pötz got his $\mathrm{Ph} . \mathrm{D}$. from the University of Graz, Austria, in 1982. His research area is the theory of semiconductors, with emphasis on optical and transport phenomena in nanostructures.

Xuedong $\mathrm{Hu}$ received his Ph.D. from the University of Michigan, Ann Arbor, in 1996. His research is in the area of squeezed phonon states and, more recently, coherent phenomena in semiconductors. 

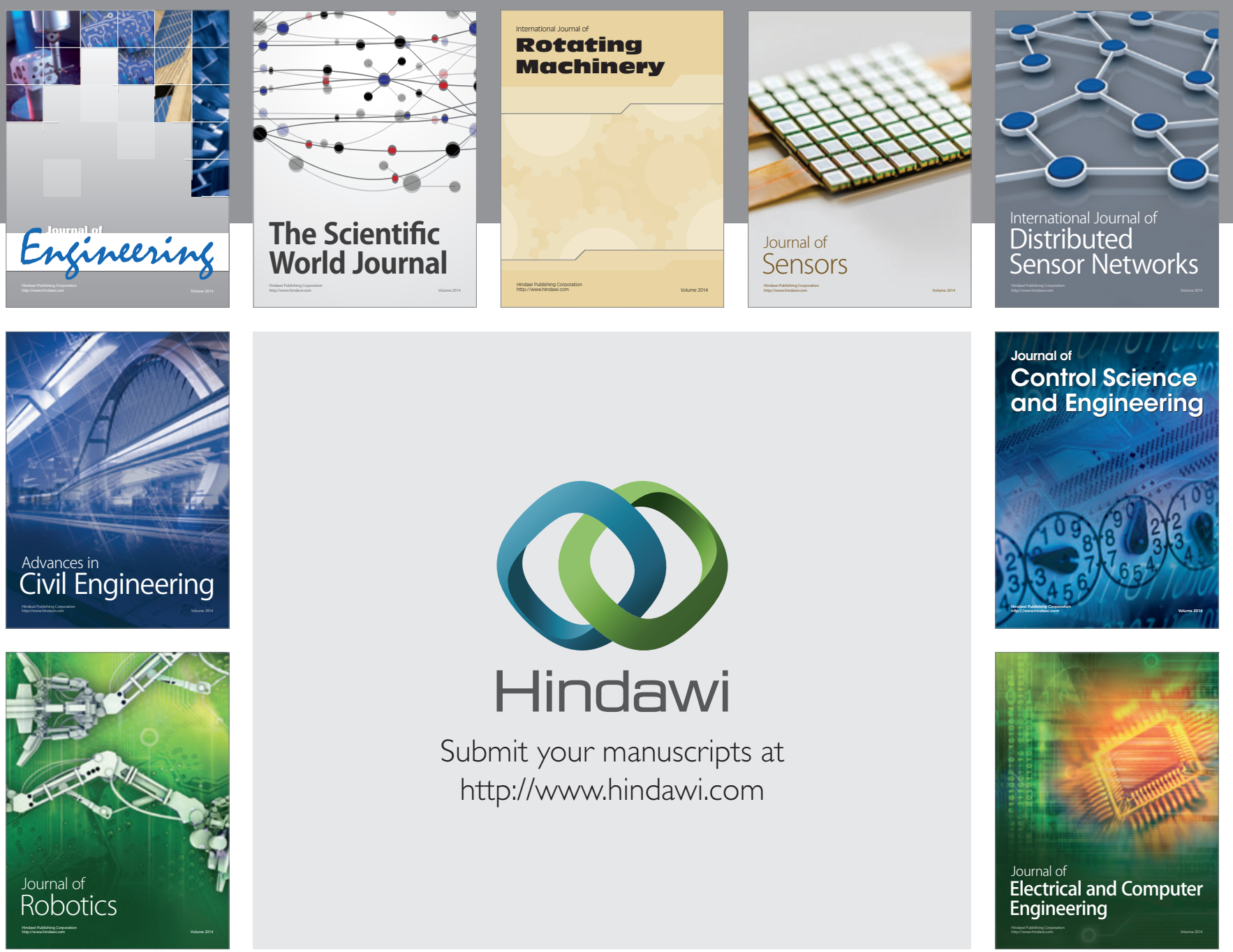

Submit your manuscripts at

http://www.hindawi.com
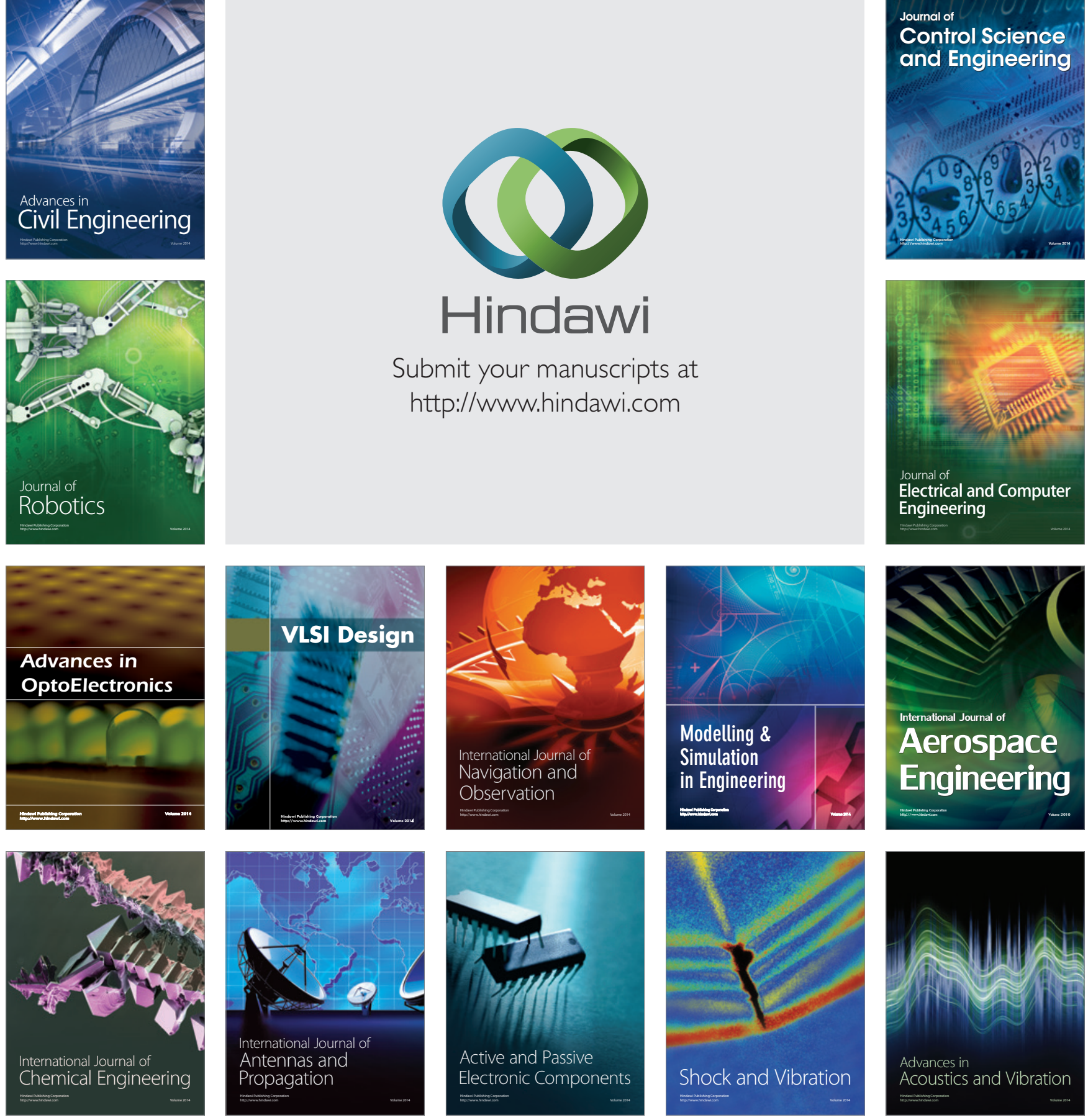